\title{
Ophthalmic nurse practitioner assessment of glaucoma: evaluating agreement within an initiative to enhance capacity in glaucoma clinics
}

\author{
Lucy Bubb $^{1} \cdot$ Divya Mathews ${ }^{1} \cdot$ Daniela Oehring ${ }^{2} \cdot$ Robert A. Harper $\mathbb{D}^{3,4}$
}

Received: 20 February 2020 / Revised: 4 December 2020 / Accepted: 5 January 2021 / Published online: 25 January 2021

(c) The Author(s), under exclusive licence to The Royal College of Ophthalmologists 2021

\begin{abstract}
Aims A local service evaluation was conducted in order to compare clinical assessment measures and management decisions between an ophthalmic nurse practitioner and a reference standard glaucoma consultant, for patients referred into secondary care with suspected Chronic Open Angle Glaucoma or Ocular Hypertension.

Methods One hundred patients were selected. A clinical pathway incorporating the assessment methods recommended by National Institute for Health and Care Excellence (NICE) Glaucoma update 2017 (NG81) was delivered by a single ophthalmic nurse practitioner and the reference standard glaucoma consultant. Clinical findings and outcomes were recorded, with both practitioners being masked to each other's findings. Agreement was determined employing Cohen's kappa, measuring inter-rater agreement allowing for chance agreement.

Results Agreement was observed as follows: Visual field assessment (kappa $k=0.806$, 95\% CI 0.661-0.951); Optical Coherence Tomography evaluation (kappa $k=0.648,95 \%$ CI 0.507-0.798); C:D Ratio assessment (Cronbach's alpha $\alpha=$ 0.96, 95\% CI 0.88-0.94); Diagnosis (kappa $k=0.874,95 \%$ CI 0.818-0.914); and Treatment planning (kappa $\kappa=0.844$, 95\% CI 0.733-0.955). In three cases the nurse practitioner judged the optic nerve to appear normal, where the reference standard examiner detected glaucoma and commenced treatment.

Conclusion This service evaluation demonstrates how an ophthalmic nurse practitioner with appropriate theoretical knowledge and practical training, can develop skills to reach a high level of agreement in patient assessment and management for those patients with suspected glaucoma. Within the limitations of a single centre and single practitioner evaluation, our findings provide evidence that this model of capacity expansion ought to merit wider consideration in secondary care glaucoma services.
\end{abstract}

Lucy Bubb

Lucy.Bubb@wales.nhs.uk

1 Department of Ophthalmology, Betsi Cadwaladr University Health Board (BCUHB) Hospital Trust, Abergele Hospital, Llanfair Road, Abergle LL22 8DP, UK

2 School of Health and Human Sciences, University of Plymouth, Plymouth, UK

3 Manchester Royal Eye Hospital and Manchester Academic Health Sciences Centre, Manchester University NHS Foundation Trust Manchester, Manchester M13 9WL, UK

4 Division of Pharmacy and Optometry, School of Health Sciences, Faculty of Biology, Medicine and Health, University of Manchester, Manchester M13 9PL, UK

\section{Introduction}

Glaucoma is one of the leading global causes of visual morbidity, potentially resulting in permanent vision loss if undiagnosed [1,2]. Detection of glaucoma and facilitation of timely glaucoma services have historically presented significant challenges within ophthalmic health care. Regrettably as we enter a new decade this predicament has burgeoned into the question of how do we provide enough capacity to meet demand, a question nevermore prescient than the era around COVID-19, with new ways of working now regarded as being key enablers in meeting these challenges.

The Royal College of Ophthalmologists Way Forward Report on glaucoma raised concerns predicating an estimated $22 \%$ rise in glaucoma over the next 10 years in the United Kingdom, with this figure set to rise annually [3]. 
It is estimated that currently 79 million people are suffering from glaucoma worldwide [4], with predictions that this figure is set to increase to a staggering 111 million by 2040 [5].

There is a multitude of published research on the successful implementation and operation of glaucoma referral filtering services, largely facilitated by optometrists in the community [6-9]. These schemes have benefited Hospital Eye Services in reducing glaucoma referrals; however, provision of timely appointments for new referrals remains a challenge, particularly within the context of an increased demand for follow-up. One of the main reasons secondary care glaucoma services are struggling to cope is due to the reported national shortage of ophthalmologists $[10,11]$. Although specialist nurses appear to be involved in glaucoma virtual clinics and glaucoma education services [12-14], there is a dearth of empirical evidence to support their involvement in an extended practical role, i.e. in accurately conducting the relevant clinical assessments and making appropriate decisions around diagnosis and monitoring. Similarly, there is a paucity of literature validating the orthoptist role in glaucoma care. This scenario contrasts to the growing literature supporting optometric glaucoma extended roles [15]. While arguably the traditional medical model still operates within many secondary care settings. Despite this paucity of published literature validating extended nursing roles, there is anecdotal evidence that such clinic models are in existence within glaucoma clinics in secondary care. Consequently, it remains vital for those employing non-medical health care professionals in advanced practice to undertake clinical research and/or clinical audit to provide evidence to underpin the safety and clinical effectiveness of multidisciplinary glaucoma care.

The National Institute for Health and Care Excellence (NICE) guideline: Diagnosis and Management of Chronic Open Angle Glaucoma (COAG) and Ocular Hypertension (OHT) (NG81), acknowledges the practicality of a multidisciplinary approach in both the principal assessment and management of these conditions [16]. Correspondingly, the Way Forward Report and Glaucoma Commissioning Guide also acknowledge the integration and delivery of new models of care to deliver sustainable ophthalmic services for the future [3, 16, 17]. In implementing these guidelines, it was proposed by the Betsi Cadwaladr University Health Board (BCUHB) Hospital Trust's Ophthalmic Department, North Wales, that new referrals with suspected COAG and OHT should be managed by one of the specialist ophthalmic nurse practitioners based at the tertiary referral centre in Abergele (Wales, UK). It was postulated that if this new model of care proved successful, this service would benefit the department by: reducing the new assessment waiting list for this patient group; facilitate the timely monitoring of glaucoma follow-up patients; and allow the glaucoma specialist consultant to manage more complex cases.

The purpose of this local service evaluation was to assess the agreement in the clinical assessment measures and management decisions of a single ophthalmic nurse practitioner and the reference standard comparator glaucoma specialist consultant, i.e. to evaluate the clinical safety of adopting this new model of care into formal longterm clinical practice. In addition, a patient satisfaction survey was undertaken to determine patients' acceptance of receiving their principal assessment with a specialist nurse practitioner in place of the traditional medical model within a consultant led glaucoma clinic. To our knowledge, service evaluation of nurse practitioner agreement with a reference standard glaucoma specialist in clinical assessment and decision making in glaucoma has not previously been reported, and such evidence is timely in consideration of support and requisite changes in practice proposed in delivering capacity in glaucoma services.

\section{Methods}

Approval from the BCUHB audit department was sought and granted to conduct the project as a local service evaluation (registered project number 17/298). The evaluation was classified as a service improvement evaluation and no ethical approval was required. The clinical evaluation took place over a period of 7 months, with data collection commencing in July 2018 and completing February 2019.

\section{Nurse practitioner training}

The nurse practitioner (LB), who was also a practising non-medical independent prescriber, had worked with the glaucoma consultant previously, completing apprentice style competency-based internal training. The glaucoma consultant (DM) trained as a glaucoma fellow before working in a substantive glaucoma specialist post at BCUHB and acts in this evaluation as the comparator reference standard. The nurse's training was focused on developing skills in optic disc assessment, anterior segment assessment, gonioscopy, and interpretation of clinical investigations including visual fields and optical coherence tomography scans. As part of the glaucoma competency assessment, the nurse carried out objectively structured clinical examinations (OSCE) which were assessed and measured as pass/fail individually by three ophthalmic consultants. These OSCEs, which had been developed by the glaucoma consultant, included assessment on the diagnosis and management of new 
glaucoma referrals, as well as assessment and monitoring of those cases previously diagnosed and under review. Together with this practical skills-based training and assessment, the nurse contemporaneously undertook a taught master's programme in Investigative Ophthalmology and Vision Science, incorporating a module dedicated to glaucoma.

\section{Participants}

All referrals from which participants were recruited had been triaged by an ophthalmologist within the ophthalmology department at BCUHB. Referrals indicative of suspected COAG or OHT were selected in consecutive and chronological order during the timeline of the evaluation, thus minimising selection bias as much as possible. Those referrals which suggested other types of glaucoma related diagnoses or non-glaucomatous pathologies or those with comorbidities were excluded. This approach was in adherence to the case mix of patients envisaged within current NICE guidance, i.e. in supporting non-medical health care professionals' involvement in assessment and diagnosis of suspected COAG and OHT [16].

\section{Patient consent}

A patient information leaflet was provided encompassing a full description of the aims of the service evaluation and individual verbal consent was sought from each patient agreeing to repeat test measures from both the nurse practitioner and glaucoma consultant. Patients were assured that confidentiality and anonymity of any service evaluation data would be applied.

\section{Clinical assessment and test methods}

The care pathway incorporated the assessment methods recommended in the updated 2017 NICE guideline: Diagnosis and Management of COAG and OHT (NG81) [16].

Standard assessment comprised of: Full medical and social history, including evaluation of symptoms; best corrected distance visual acuity assessment for both eyes (LogMAR); SITA 24-2 threshold visual field test (Zeiss Humphrey visual field analyser, Carl Zeiss Meditec AG, Germany); Optical Coherence Tomography to assess retinal nerve fibre layer (RNFL) and posterior pole (Heidelberg Spectralis OCT, Germany); slit-lamp bio-microscopy examination for assessment of the anterior segment of both eyes (Haag-Streit International); Intraocular pressure assessed using Goldmann applanation technique (HaagStreit, Switzerland); central corneal thickness measurement (DGH Pachmate 2 pachymeter); gonioscopic examination to assess drainage channels (Ocular Magnaview gonioscopy lens, employing Shaffer grading classification); optic nerve head assessment using slit-lamp biomicroscope and Volk $+60 \mathrm{D}$ non-contact lens with pupil dilation (Tropicamide $1 \%$ ); wider fundus examination using slit-lamp biomicroscope and Volk +90D lens; and fundus photography (Topcon TRC NW8F Retinal camera. Topcon Medical Systems. INC, Tokyo, Japan).

\section{Quality of service patient questionnaire}

A service questionnaire was distributed to each patient during the initial assessment, with consent being obtained to use anonymised patient satisfaction responses and comments to evaluate the experience received. The survey was designed to assess patients' acceptance of receiving their principal appointment with the specialist nurse practitioner rather than medical staff. Questions included: Did the nurse introduce herself and explain what to expect during your appointment; were examination techniques delivered confidently; and were you happy to see a specialist nurse for your initial assessment today? Six closed questions (yes, no, unsure) were used to reduce the response burden and encourage a high response rate. It was anticipated that due to the repeat test measures resulting in longer consultations, as well as having dilated pupils necessary for examination, participants may be less inclined to complete a survey containing open questions. However, a comment box was made available for those who wished to offer further feedback on their experiences. Participants were instructed to leave the completed survey in a designated postage box within the reception area on completion of their appointment.

\section{Diagnostic tests}

Patients with adequate physical and mental capacity performed a Humphrey 24-2 SITA standard test to assess their visual field. Visual field outcomes were independently classified by the two practitioners into one of the following categories: No defect, a suspected defect/borderline changes; and a definitive glaucomatous defect. For imaging, both practitioners again independently interpreted the output from the OCT RNFL measures into one of the following categories: within normal limits; glaucoma suspect; or definitive glaucomatous damage being evident. Following these test measures, a complete medical and social history was acquired on each patient, including any risk factors associated with glaucoma.

\section{Clinical examination}

The nurse practitioner, who was trained in external and internal eye examination methods, examined the anterior segment via slit-lamp bio-microscopy, incorporating measuring intraocular pressure with GAT and CCT of both 
eyes. These latter measures were not included in the agreement comparison to avoid unnecessary patient burden, i.e. in those glaucoma related skills where the nurse's prior clinical experience and satisfactory OSCE assessments did not necessitate repeat testing by the reference standard.

Following anterior segment examination, gonioscopy was performed by both practitioners, with the Shaffer grading system being documented in the clinical notes, albeit for the purposes of the comparison made, gonioscopic outcomes are provided in the evaluation results as open, narrow or closed angle. Where cases were deemed to have sufficiently open angles, both pupils were dilated for a fundus examination, including assessment of the optic nerve and photography of the optic discs. The global measure of optic disc status (the Cup to Disc Ratio, CDR), was graded between 0.00 and 1.00 in each eye separately. For all parts of the anterior segment and posterior segment examination, each practitioner independently recorded their own measures masked to each other's findings.

\section{Clinical assessment and decision making}

Following diagnostic testing and clinical examination, the glaucoma consultant and nurse practitioner independently formulated a diagnosis and clinical management plan intended to best meet the needs of each individual patient. Pre-defined categories for diagnoses were agreed prior to commencement of the evaluation, and included: Normal, OHT, glaucoma suspect, glaucoma, optic disc asymmetry; and other (to include a differential diagnosis). Although not a definitive diagnosis, optic disc asymmetry was included due to the evidence linking it as a risk factor to COAG [3]. consequently, these individuals would necessitate further assessment in the future. Those individuals believed to require intraocular pressure lowering treatment were commenced on a prostaglandin analogue (Latanoprost $0.005 \%$ ) where not contraindicated, subsequently these cases were listed for a 6-week review to assess their adherence, tolerance and the effectiveness of commencing this treatment. The remaining management outcome categories included: Glaucoma suspects (6-9 months review); OHT, not treated (as per NICE guidance discharged for monitoring by community optometrist); non-glaucomatous differential diagnosis (referred to appropriate specialist); and no suspicion of glaucoma or co-morbidity necessitating management (discharged). During the timeline of the evaluation the final decision on patient management resided with the reference standard glaucoma consultant.

\section{Statistical methods and analysis}

One hundred patients were examined, with the results being collected for both eyes, since glaucoma can be a unilateral eye disease and therefore, overall, both practitioners assessed 200 eyes. Due to varying patient circumstances, for example an inability to comply with the demands of testing, not all assessments were able to be completed for all 100 patients, and therefore, a reduced sample size was available for some of our analyses. One eye per patient was randomly chosen for inclusion in our analyses (50\% right eyes, 50\% left eyes), since analysing such a sample provides a more precise depiction of the inter-observer agreement between practitioners, i.e. due to the strong possibility of inter-eye correlations in each pair of eyes [18, 19]. Data analysis was completed by a statistician located remotely, with no prior knowledge of patient demographics, measures taken nor decisions made by either the glaucoma consultant or nurse practitioner. Data was collated and analysed in Excel 2016 and R for Mac (R1.71). Standard descriptive analysis was employed. Inter-observer agreement between the nurse practitioner and the reference standard was assessed for all outcome measures using Cohen's kappa for nominal data and weighted kappa for ordinal data with linear weighting, with the alternative hypothesis being that the agreement between the two examiners is different to chance agreement. Weighted kappa with linear weighting was used since the size of the outcome differences were important, but it was assumed that it was additive not multiplicative. Inter-observer values were classified as: $>0.75$ indicates excellent agreement, $0.40-0.75$ intermediate agreement and $<0.40$ poor agreement [20].

\section{Results}

\section{Patient classification}

Patients included in the evaluation had a mean age of $68 \pm$ 12 years (range 39-93 years), with $51 \%$ being female and $49 \%$ male. The primary reason for referral of cases was the suspicion of COAG (46.5\%), or OHT (18.8\%). Other referrals reasons included: Evidence of disc haemorrhage (11.9\%); optic disc asymmetry/suspicious looking nerve $(8.0 \%)$; visual field defect $(5.0 \%)$; and family history of glaucoma $(4.0 \%)$.

\section{Agreement analysis for measures of structure, function and clinical assessment}

\section{Visual fields (practitioners' SITA 24-2 outcome classifications)}

There was excellent agreement between examiners, with the overall percentage agreement being 92.9\% (Weighted Cohen's $\kappa=0.806,95 \%$ CI 0.661-0.951), (Table 1). 


\section{OCT analysis (RNFL and posterior pole)}

The overall percentage agreement between the two examiners was $78.6 \%$. There was moderate intra-observer agreement (Weighted Cohen's $\kappa=0.648,95 \%$ CI 0.507 , 0.798), (Table 2).

\section{Gonioscopy}

Weighted Cohen's $\kappa$ was used to determine if there was agreement between two examiners on angle assessment in 57 eyes being rated as either open, narrow, or closed. The overall percentage agreement between the two examiners is $100 \%$ $(\kappa=1.000)$, (Table 3$)$. Note that the aim of the evaluation was to assess only those cases with suspected COAG or OHT. It was agreed the nurse practitioner would continue this assessment for the remainder of the study, hence the smaller sample included and the need for caution in interpretation, given the largely open angle status of the case mix included.

\section{Optic disc summary measure (CDR)}

A total of 98 eyes were included in the CDR comparison. The median CDR assessed by the nurse practitioner and the glaucoma specialist was found to be 0.6 (95\% CI 0.6-0.7). Cronbach's alpha was found to be excellent $(0.96,95 \%$ CI 0.88-0.94), although as noted below there were instances of apparent underestimation of neuro-retinal rim loss (see agreement in diagnosis and treatment planning) not

Table 1 Visual fields (practitioners' HFA 24-2 outcome classifications).

\begin{tabular}{llllll}
\hline & & \multicolumn{3}{l}{ Glaucoma specialist } & \\
\cline { 3 - 6 } & & No defect & Borderline & Defect & Total \\
\hline $\begin{array}{l}\text { Nurse } \\
\text { practitioner }\end{array}$ & No defect & 76 & 2 & 2 & 80 \\
& Borderline & 1 & 7 & 0 & 8 \\
& Defect & 1 & 1 & 9 & 11 \\
& Total & 78 & 10 & 11 & 99 \\
\hline
\end{tabular}

Visual fields (practitioners' HFA 24-2 outcome classifications) N 99: percentage agreement $92.9 \%$. $(\kappa=0.806,95 \%$ CI $[0.661,0.951])$ (Table 1). captured by summary comparison of the global CDR measures between examiners.

\section{Agreement in diagnosis and treatment planning}

There was an excellent level of agreement statistically between practitioners in both diagnosis and treatment planning decisions. The overall percentage agreement in diagnosis was $87.9 \%(\kappa=0.874,95 \%$ CI $0.818,0.914)$, (Table 4). For treatment planning the overall agreement was $92.9 \%(\kappa=0.844,95 \%$ CI $0.733,0.955)$, (Table 5). The discrepancies in diagnosis and treatment planning are as follows: In four cases the nurse practitioner judged the optic nerve to appear glaucomatous, consequently opting to start treatment in three of these cases. Of these four cases the outcomes were as follow: In one case the glaucoma consultant offered the option of treatment or monitoring, to which the individual chose monitoring; in a further two cases the glaucoma consultant judged physiological cupping, but with a degree of suspicion to necessitate followup; and in the final case, while there was agreement on appearance of the optic nerve, the glaucoma specialist preferred a watch and wait review versus that of commencing treatment. In three cases the nurse practitioner judged the optic nerve to appear normal, where the reference standard examiner detected glaucoma and commenced treatment. On further inspection it was established in these cases the nurse practitioner failed to identify thinning of the neuro-retinal rim or had reduced recognition of increased, but shallow cupping.

Table 3 Gonioscopy.

\begin{tabular}{llllll}
\hline & & \multicolumn{4}{l}{ Glaucoma specialist } \\
\cline { 3 - 6 } & & Open & Narrow & Closed & Total \\
\hline Nurse practitioner & Open & 55 & 0 & 0 & 55 \\
& Narrow & 0 & 2 & 0 & 2 \\
& Closed & 0 & 0 & 0 & 0 \\
& Total & 55 & 2 & 0 & 57 \\
\hline
\end{tabular}

Gonioscopy: N 57; percentage agreement between the two examiners $100 \%$. $(\kappa=1.000)$ (Table 3$)$.
Table 2 OCT analysis (retinal nerve fibre layer and posterior pole).

\begin{tabular}{llllll}
\hline & \multicolumn{4}{l}{ Glaucoma specialist } \\
\cline { 3 - 6 } & & Normal & Glaucoma suspect & Glaucoma & Total \\
\hline \multirow{2}{*}{ Nurse practitioner } & Normal & 56 & 4 & 1 & 61 \\
& Glaucoma suspect & 1 & 20 & 10 & 31 \\
& Glaucoma & 2 & 3 & 1 & 6 \\
& Total & 59 & 27 & 12 & 98 \\
\hline
\end{tabular}

OCT analysis (retinal nerve fibre layer and posterior pole) $\mathrm{N} 98$; percentage agreement $78.6 \%$. $(\kappa=0.648$, 95\% CI [0.507, 0.798]) (Table 2). 
Table 4 Agreement in diagnosis.

\begin{tabular}{|c|c|c|c|c|c|c|c|c|}
\hline & & \multicolumn{7}{|c|}{ Glaucoma specialist } \\
\hline & & Normal & Disc asymmetry & OHT & Glaucoma Suspect & Glaucoma & Differential diagnosis & Total \\
\hline \multirow[t]{7}{*}{ Nurse practitioner } & Normal & 37 & & & & 3 & 1 & 41 \\
\hline & Disc asymmetry & 1 & 8 & & 1 & & & 10 \\
\hline & $\mathrm{OHT}$ & 1 & & 1 & & & & 2 \\
\hline & Glaucoma Suspect & & & & 16 & 1 & & 17 \\
\hline & Glaucoma & & & & 4 & 22 & & 26 \\
\hline & Differential diagnosis & & & & & & 3 & 3 \\
\hline & Total & 39 & 8 & 1 & 21 & 26 & 4 & 99 \\
\hline
\end{tabular}

Agreement in diagnosis: N 99; percentage agreement $87.9 \%(\kappa=0.874,95 \%$ CI $[0.818,0.914])$ (Table 4).

(Differential diagnosis: anterior ischemic neuropathy (AION) and retinal pathology).

Table 5 Treatment planning.

\begin{tabular}{lllll}
\hline & & \multicolumn{3}{l}{ Glaucoma specialist } \\
\cline { 3 - 5 } & & Yes & No & Total \\
\hline Nurse practitioner & Yes & 31 & 3 & 34 \\
& No & 4 & 61 & 65 \\
& Total & 35 & 64 & 99 \\
\hline
\end{tabular}

Treatment planning: $\mathrm{N} 99$ percentage agreement is $92.9 \%$. $(\kappa=0.844$, $95 \%$ CI $[0.733,0.955])$ (Table 5).

\section{Quality of service patient survey}

A total of 73 patients completed the quality of service survey, all of whom indicated their satisfaction with the service and their acceptance of being assessed by the specialist nurse practitioner. For the question about examination techniques being delivered confidently, all 73 answered yes and all responded that they were happy to see a specialist nurse for their initial assessment on the day, with four patients adding to the open field comments option as follows: 'Staff as usual all wonderful. God bless you all'; 'The whole process is most professional and carried out in a friendly manner'; ' $100 \%$ happy with the overall service'; 'My only complaint is long waiting time from time of arrival, which is frustrating'.

\section{Discussion}

This service evaluation is the first study to provide evidence of a high level of agreement between a specialist ophthalmic nurse practitioner and a sub-specialist glaucoma consultant in the assessment and management of new referrals to secondary care for suspected COAG or OHT. However, it should be acknowledged that this local evaluation is from a single centre and with a single ophthalmic nurse practitioner, limiting generalisability. While the overall level of agreement was high, complete agreement in all areas of assessment was not achieved and notably there were a small number of cases where diagnostic and treatment decisions differed between the practitioners. Furthermore, there is incomplete evidence around the specific skill of gonioscopy due to the nature of cases included in this specific evaluation. The disparity was predominantly in the categorisation of definitive glaucoma versus glaucoma suspect status, with the nurse practitioner arguably over diagnosing those cases where the reference standard glaucoma consultant had classified patients as glaucoma suspect; however, it is well documented that the diagnosis of glaucoma, the evaluation and semi-quantification of the optic nerve and interpretation of glaucoma diagnostic tests (notably interpretation of OCT and visual fields) can be fairly subjective and inter-observer variability is often significant [21-23]. Although classification differed in selected cases of over diagnosis, there was little or no difference in the CDR assessments of the optic nerve in these instances. Otherwise, there was full agreement on a further 22 eyes considered to be glaucomatous. Significantly, however, in three eyes the nurse practitioner determined a normal diagnosis where the reference standard decision was a diagnosis of glaucoma. These three cases can be considered false negatives, albeit at a low rate of $3 \%$ in this sample. On case reviewing, it was established that the nurse practitioner had failed to identify neuro-retinal rim thinning, and in another case, there was a failure in the identification of a shallow sloping cup, which is, arguably notoriously challenging to assess. In these instances of more significant difference, it may have proved beneficial to have had a third person acting in arbitration, albeit the design of the service evaluation here had the consultant as the reference standard against which the nurse practitioner was being compared. Nevertheless, it must be conceded that the absence of arbitration is a limitation of this study, not least within the context of the extent to which experts can and do disagree in glaucoma assessment and management. Indeed, there is abundant literature available on 
the challenge practitioners face in diagnosing glaucoma [2124]. This challenge is due in part to the complex multi factorial nature of the disease, as well as other conditions, both ophthalmic and systemic, resulting in damage to the optic nerve and/or visual field. A systematic approach must be followed in all aspects of assessment, from clinical history taking (which may identify risk factors associated with glaucoma), to the assessment of clinical test measures, and rigorous evaluation of the optic nerve. While there is a wealth of resources available to aid practitioners in their professional development, arguably ongoing clinical experience through working alongside medical practitioners to gain 'hands on experience' and exposure to the diversity of authentic clinical cases is central to improvements in practice for both nonmedical health care practitioners and medical practitioners in training. Indeed, there is some evidence for the impact of such experience and the taking of additional glaucoma qualifications, for example, in better performance in optic disc assessment [25].

The high response rate and positive outcomes of the patient satisfaction survey offers evidence of the acceptance of a new model of care provided by non-medical health care professionals, a finding that is in keeping with available literature corroborating acceptance of nurse practitioners in advancing care roles [26, 27].

There is unanimous agreement in the United Kingdom that ophthalmic care and the maintenance of eye health is paramount $[3,11,16,17,28]$. The Royal College of Ophthalmologists workforce census in 2018 reported that $40 \%$ of eye departments were dependent on utilising non-medical health care professionals in advanced practice [11], furthermore, noting that the smaller rural units are where the vacant ophthalmology positions predominate, and therefore, where non-medical health care professionals are relied upon to sustain services [11]. Ophthalmology has the prospect of being a pioneering speciality affording the employment of an array of specialist practitioners. Whilst these practitioners cannot replace the expertise of medical staff, they can complement the medical team in delivering holistic care and ensuring people are seen by the most suitable person to meet their needs. With predictions reporting increased demand for glaucoma care to be rising annually [3], developing sustainable services is paramount to prevent avoidable sight loss. There are numerous published studies demonstrating the performance of optometrists in extended roles within glaucoma $[1,6-9,24]$. However, there is a paucity of quantifiable evidence on agreement in the clinical assessment and decision making underpinning the effectiveness of nurse practitioners working in these extended roles, with the authors identifying only one previously published study in the peer reviewed literature, a study restricted to evaluating performance of nurse practitioners with tonometry [29]. The present service evaluation, with limitations acknowledged, does provide empirical evidence of the expertise nurse practitioners may bring to glaucoma services. Although it has been acknowledged there were discrepancies in a small number of cases, overall the level of agreement in both clinical assessment and clinical decisions was high. Indeed, as a result of this evaluation the BCUHB Trust has continued employing the nurse practitioner within the role described herein, working alongside the glaucoma consultant in the assessment of new patients with suspected COAG or OHT.

In conclusion, this service evaluation demonstrates that a specialist nurse practitioner, with appropriate theoretical knowledge, practical training, and working within a supportive environment under the supervision of a glaucoma consultant, is able to develop their skills to reach a high level of agreement in patient assessment and diagnosis set against the reference standard glaucoma consultant. This evaluation, while limited to a single practitioner in a single centre, displays proof of concept that this approach to capacity enhancement can be a suitable model, where locally required, adding further to the existing evidence around specialist non-medical health care professional roles being used to meet the rising demand in glaucoma care.

\section{Summary}

\section{What was known before}

- Growth in elderly population resulting in inevitable increase eye diseases such as glaucoma.

- New models of care are currently necessary if we are to cope with capacity to meet demand in the future.

- Specialty trained optometrists have developed their expertise to advance practice in glaucoma care. However, there is a paucity of literature supporting ophthalmic nurses in these roles.

\section{What this study adds}

- With medical support and training, specialist ophthalmic nurse practitioners are able to reach a high level agreement against the reference standard glaucoma specialist in the diagnosis and management of COAG and OHT.

- A new model of glaucoma service design can form part of capacity expansion in secondary eye care services.

Acknowledgements We would like to thank the Betsi Cadwaladr University Hospital Trust for allowing us to conduct this service evaluation study, and a special thank you to all participating patients. 
Author contributions The analysis and interpretation of the data was undertaken initially by LB and DO, joined subsequently by RAH and DM. The paper was drafted by LB with RAH and revised by DM and DO. All authors contributed to the design of the study and approved the final version of the paper.

\section{Compliance with ethical standards}

Conflict of interest The authors declare that they have no conflict of interest.

Publisher's note Springer Nature remains neutral with regard to jurisdictional claims in published maps and institutional affiliations.

\section{References}

1. Azuara-Blanco A, Burr J, Thomas R, Maclennan G, Mcpherson S. The accuracy of accredited glaucoma optometrists in the diagnosis and treatment recommendation for glaucoma. $\mathrm{Br} \mathrm{J}$ Ophthalmol. 2007;91:1639-43.

2. Quigley HA. Glaucoma. Lancet. 2011;377:1367-77.

3. The Royal College of Ophthalmologists. The way forward: options to help meet demand for the current and future care of patients with eye disease: glaucoma. London: The Royal College of Ophthalmologist; 2017.

4. Quigley HA, Broman A. The number of persons with glaucoma worldwide in 2010 and 2020. Br J Ophthalmol. 2006;90:151-6.

5. Tham YC, Li X, Wong TY, Quigley HA, Aung T, Cheng CY. Global prevalence of glaucoma and projections of glaucoma burden through 2040: a systematic review and meta-analysis. Ophthalmology. 2014;121:2081-90.

6. Spry PG, Spencer IC, Sparrow JM. The bristol shared care glaucoma study: reliability of community optometric and hospital eye service test measures. Br J Ophthalmol. 1999;83:707-12.

7. Parkins DJ, Edgar DF. Comparison of the effectiveness of two enhanced glaucoma referral schemes. Ophthalmic Physiol Opt. 2011;31:343-52.

8. Henson DB, Spencer AF, Harper R, Cadman EJ. 'Community refinement of glaucoma referrals. Eye. 2003;17:21-6.

9. Devarajan N, Williams GS, Hopes M, O'Sulliven D, Jones D. The carmarthenshire glaucoma referral refinement scheme: a safe and efficient screening service. Eye. 2011;25:43-9.

10. The Royal College of Ophthalmologists. Workforce census 2016. London: Royal College of Ophthalmologists. http://www.rcophth. ac.uk/publications/workforce-census-2016.

11. The Royal College of Ophthamologists. Workforce census 2018. London: Royal College of Ophthalmologists. http://www.rcophth. ac.uk/publications/workforce-census-2018.

12. Lee H. Royal National Institute for Blind People. Expanding community eye care for glaucoma: a pilot ophthalmic diagnostic and treatment centre. Cymru: RNIB; 2015 - revised February 2016. https://www.rnib.org.uk/expanding-community-eye-care.

13. Vernon SA, Adair A. Shared care in glaucoma: a national study of secondary care lead schemes in England. Eye. 2010;24:265-9.

14. Ney JJ. Glaucoma diagnosis and treatment: the role of the ophthalmic nurse. Insight. 2016;41:13-7.

15. Harper RA, Gunn P, Spry PGD, Fenerty CH, Lawrenson JG. Care pathways for glaucoma detection and monitoring UK. Eye. 2019;34:89-102.

16. National Institute for Health and Care Excellence. (2017). Glaucoma: diagnosis and management guideline (NG81). http://www. nice.org.uk/guidance/NG81.

17. The Royal College of Ophthalmologists Commissioning Guide: 'Glaucoma and Ocular Hypertension. Commissioning guide': glaucoma. London: Royal College of Ophthalmologists and the Clinical Council for Eye Health Commissions; 2016.

18. Rosner B. Statistical methods in ophthalmology: an adjustment for the interclass correlation between eyes. Biometrics. 1982;1: $105-14$.

19. McAlinden C, Khadka J, Pesudovs K. Statistical methods for conducting agreement (comparisons of clinical tests) and precision (repeatability or reproducibility) studies in optometry and ophthalmology. Ophthalmic Physiol Opt. 2011;4:330-8.

20. Fleiss JL, Levin B, Paik MC. Statistical methods for rates and proportions (3rd ed). Hoboken, NJ: Wiley; 2003.

21. Varma R, Steinmann WC, Scott I. Expert agreement in evaluating the optic disc for glaucoma. Ophthalmology. 1992;99:215-21.

22. Fingeret M. Glaucoma and guided progression analysis-how can clinicians differentiate disease progression from normal variability in visual fields. Ophthalmic Prof. 2014;3:28-9.

23. Johnson ZK, Siddiqui R, Azuara-Blanco A. The quality of reporting of diagnostic accuracy studies of optical coherence tomography in glaucoma. Ophthalmology. 2007;114:1607-12.

24. Ho S, Vernon SA. Decision making in chronic glaucomaoptometrists vs ophthalmologists in a shared care service. Ophthalmic Physiol Opt. 2011;31:168-73.

25. Hadwin SE, Redmond T, Garway-Heath DF, Lemij HG, Reus NJ, Ward G, et al. Assessment of Optic Disc Photographs for Glaucoma by UK Optometrists: The Moorfields Optic Disc Assessment Study (MODAS). Ophthalmic Physiol Opt. 2013;33: 618-24.

26. Mitchell T, Butler-William C, Easton K, Ingledew I, Parkin D, Wade $\mathrm{S}$, et al. The consultant nurse expert practitioner and so much more. Br J Nurs. 2010;8:481-8.

27. Sustaita A, Zeigler V, Brogan M. Hiring a nurse practitioner: what's in it for the physician?. Nurse Pract. 2013;11:41-5.

28. Royal National Institute of Blind people \& Specsavers. The State of the Nation Eye Health 2017: A Year in Review. Transforming eye health care. https://www.rnib.org.uk/state-nation- 2017.

29. Kotecha A, Elkarmouty A, Ajtony C, Barton K. Interobserver agreement using Goldmann applanation tonometry and dynamic contour tonometry: comparing ophthalmologists, nurses, and technicians. Br J Ophthalmol. 2015;100:854-9. 